\title{
SISCI - SISTEMA PARA CONTROLE DE IRRIGAÇÃO ATRAVÉS DE DISPOSITIVOS CELULARES
}

\author{
L. I. de Almeida ${ }^{1}$, I. F. C. Fernandes ${ }^{1}$ e C. M. Costa ${ }^{1}$ \\ ${ }^{1}$ Departamento de Informática - Instituto Federal do Rio Grande do Norte \\ \{leandromoreno, islamefelipe\}@hotmail.com - clayton.maciel@ifrn.edu.br
}

Artigo submetido em setembro/2011 e aceito em fevereiro/2012

\section{RESUMO}

O advento das tecnologias de informação, a expansão da Web e as modernas necessidades de mobilidade e compartilhamento de dados tiveram como resultado o investimento em sistemas adequados a dispositivos móveis, como o aparelho celular. Entretanto, utilização dessas tecnologias para otimização de processos pertinentes à área agrícola é pouco utilizada, e o desenvolvimento do projeto $\mathrm{Sis} \mathbf{C l}$ baseia-se nessa necessidade. Esse projeto trata-se do desenvolvimento de um sistema de tempo real para o controle e monitoramento remoto de irrigações através de celulares com acesso a Internet. Tem por objetivo introduzir novas tecnologias às irrigações da região do
Vale do Açu, provendo redução de custos para os proprietários agrícolas. Atualmente, o projeto está sendo implementado em uma fazenda escola da rede federal de ensino técnico e superior, localizada no município de Ipanguaçu no estado do Rio Grande do Norte. Por fim, o projeto tem como principais resultados: (i) o sistema $\mathrm{SisCl}$ com documentação e código executável seguindo a arquitetura padrão W3C para serviços Web; (ii) três trabalhos de conclusão de curso técnico; (ii) três artigos e três banners para SEATEC e CONNEPI; e, (iv) cursos para a comunidade de Ipanguaçu.

PALAVRAS-CHAVE: Irrigação, Monitoramento Remoto, Sistema Web de Tempo Real, Dispositivo Móvel.

\section{SISCI - IRRIGATION CONTROL SYSTEM THROUGH MOBILE DEVICES}

\section{ABSTRACT}

The advent of information technologies, the growth of Web and the new conceptions of mobility and sharing data resulted in investment in systems for mobile devices, as mobile phone. However, these relevant technologies are few used to optimization processes in the agricultural area, and the development of this work is based on this need. The $\mathrm{SisCl}$ project consists of a real time system for control and remote monitoring of irrigation systems through mobile phones on the Web. This work introduces new irrigation technologies in the Vale do Açu region, providing cost savings to the agricultural owners. Currently, the project is being applied at a School Farm of the Federal Institute of Technical and Higher Education, located in city of Ipanguaçu in the state of Rio Grande do Norte. The main results of this work are: (i) the $\mathrm{SisCl}$ system with your documentation and your implementation that follow the W3C standard architecture for Web Services; (ii) three conclusion work of computer technician course; (iii) three papers and three banners for SEATEC and CONNEPI; and, (iv) courses for the community Ipanguaçu.

KEY-WORDS: Irrigation, Remote Monitoring, Real Time Web System, Mobile Device. 


\section{SISCI - SISTEMA PARA CONTROLE DE IRRIGAÇÃO ATRAVÉS DE DISPOSITIVOS CELULARES}

\section{INTRODUÇÃO}

A expansão da Web, o advento das novas tecnologias de informação e comunicação, e a necessidade de compartilhamento de dados com alta disponibilidade, somada à avançada tecnologia de dispositivos móveis a custos baixos, motivaram o desenvolvimento de sistemas para dispositivos móveis (celulares, smartphones, PDAs e etc.) em diversas áreas.

Um dispositivo móvel cada vez mais popular em todo o mundo tem sido o telefone celular. O uso da telefonia celular cresce a cada dia em todas as partes do mundo, quer seja para trabalho, estudo, entretenimento ou para outras tantas atividades comuns do dia a dia. A prova disso é a estimativa de que, no mundo, existam aproximadamente 4,6 bilhões de assinaturas de telefones celulares, e esse número pode chegar aos cinco bilhões antes mesmo do fim do ano de 2011. Dessa forma, pode-se alcançar a incrível marca de um telefone celular para cada 1,36 pessoas no mundo (SZAMES, 2010).

Assim, uma excelente forma de aproveitar esses avanços tecnológicos e sociais pelos quais passa o mundo nas últimas décadas, tem sido investir pesquisa sobre sistemas adequados a celulares. As aplicações em dispositivos celulares podem ser usadas em qualquer lugar, o que garante a disponibilidade de acesso a informações e a mobilidade. Atualmente, a utilização dos dispositivos celulares tem sido empregada em diversas áreas, como em sistemas bancários, sistemas de controle de tráfego aéreo, sistemas de monitoramento na área médica e petrolífera, sistemas de manufatura, sistemas de sensoriamento eletrônico, sistemas de identificação da polícia federal e etc (DONG, 2006; WEI-MIN, 2006; COSTA, 2009; COSTA, 2008 e SALIM, 2004). Sendo assim, é percebível que o uso de dispositivos celulares traz inúmeros benefícios para as aplicações, entre eles, a alta disponibilidade, mobilidade e escalabilidade de informações.

Na área agrícola percebe-se apenas a utilização de sistemas de monitoramento tradicionais para otimização de processos, controle/manejo de irrigação, geração de relatórios, identificação e alerta de pragas. Esses sistemas, se comparados aos atuais avanços tecnológicos, além de arcaicos apresentam alto custo de implantação e utilização tanto para o proprietário como para o meio ambiente e/ou não utilizam tecnologias Web. Sendo assim, a maioria dos sistemas agrícolas não se aproveita do leque de benefícios da tecnologia da informação, e tão menos da utilização dessa tecnologia aplicada em dispositivos celulares. Nesse sentido, a utilização de dispositivos celulares para controle de irrigação contribui para irrigações mais eficazes e menos dispendiosa para os proprietários agrícolas, técnicos, engenheiros dessa área e etc. Isso já que uma grande vantagem da utilização dessa tecnologia nessa área é, por exemplo, a potencial diminuição da preocupação do proprietário em estar diretamente "no campo" para manipular/monitorar a irrigação de seu plantio.

É com base nessas necessidades que se motiva o desenvolvimento do projeto SisCl, um produto livre de licença e que trabalha com tecnologias atuais, um sistema com processos automatizados para irrigação de plantação(ões) que consiste em especificar e implementar um sistema de tempo real para o controle e monitoramento remoto de irrigação(ões) através de dispositivos celulares. A solução abrange culturas, jardins e gramados, que podem ser irrigados em dias e horários pré-programados, com a duração de tempo determinado para 
atender às necessidades específicas de cada área e do tipo de vegetação. Depois de completamente implantado, o sistema cessa a preocupação com a rega, pois tal serviço é monitorado e executado automaticamente.

O projeto SisCl tem ainda, uma função extensora, com o fim de promover a interação entre a Fazenda Escola (campus Ipanguaçu) e a sociedade, isso através de oficinas e minicursos ministrados pelos próprios bolsistas e voluntários do projeto em eventos da instituição e nas escolas públicas do município de Ipanguaçu. Além disso, com a perspectiva interdisciplinar entre Informática, Eletrônica e Agroecologia, o projeto proposto amplia a capacidade de seus autores e interlocutores em trabalhar com novas tecnologias, posicionarse diante da informação e interagir de forma crítica e ativa, no meio físico e social, contribuindo para que eles aprimorem de forma valiosa os seus conhecimentos. A seguir, é exposto em detalhes todo o funcionamento do projeto $\mathrm{Sis} \mathrm{Cl}$.

\section{FUNDAMENTAÇÃO TEÓRICA}

\section{Sistemas de Tempo Real}

Os STRs (Sistemas de Tempo Real) são sistemas que reagem a estímulos oriundos de um ambiente dentro de prazos específicos. Comumente, são inerentes a aplicações críticas, caracterizadas por geralmente envolverem o risco de vidas humanas, perdas econômicas ou até mesmo danos ao meio ambiente (RIBEIRO NETO, 2004).

Um STR consiste, basicamente, em um sistema controlador e um sistema controlado, como ilustra a Figura 1. O sistema controlado é um ambiente teoricamente externo ao sistema controlador e disponibiliza dados para este. E é com base nos dados vindos do sistema controlado, que o sistema controlador trabalha sobre ele. Os dados oriundos do sistema controlado podem ser obtidos de um sensor ou de uma rede de sensores que esteja implantada nesse sistema e colhe dele os dados necessários; uma vez que os sensores absorvem informações, eles as enviam para o sistema controlador. Em sistemas críticos é indispensável que o sistema controlador obtenha informações sobre o estado real do ambiente de forma que os resultados obtidos sejam os esperados, a confiabilidade do sistema deve ser impecável, pois de outra forma, podem ser gerados resultados catastróficos.

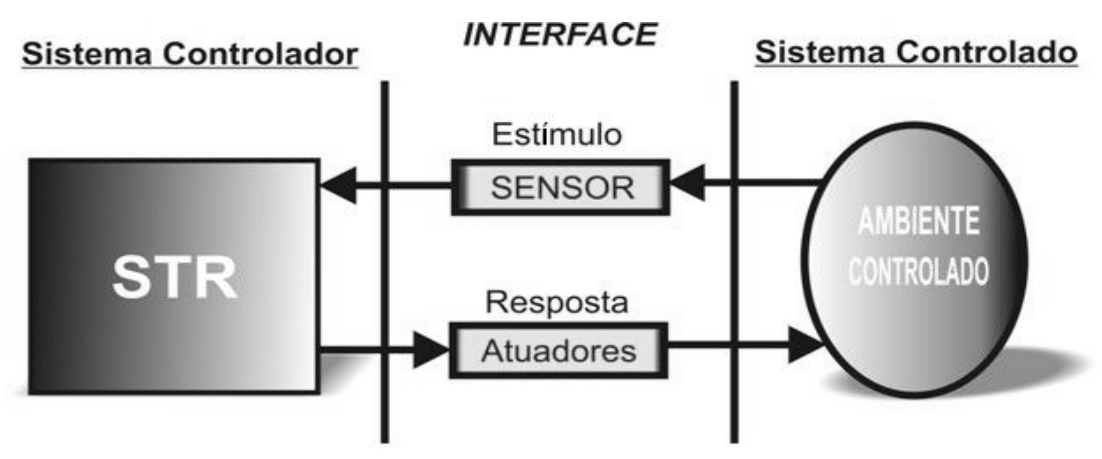

Figura 1 - Arquitetura de um STR 
Geralmente, os dados colhidos e emitidos pelos sensores são dados com restrição de tempo, por isso não dependem apenas dos resultados lógicos em si, mas também do tempo em que os resultados são produzidos. Um exemplo muito fácil de um STR é um sistema de freios computadorizado para automóveis, ilustrado na Figura 2. Esse sistema é composto de um atuador, um sensor de velocidade. O freio no pneu do automóvel tem que ser acionado numa restrição de tempo quando um usuário pisa no pedal.

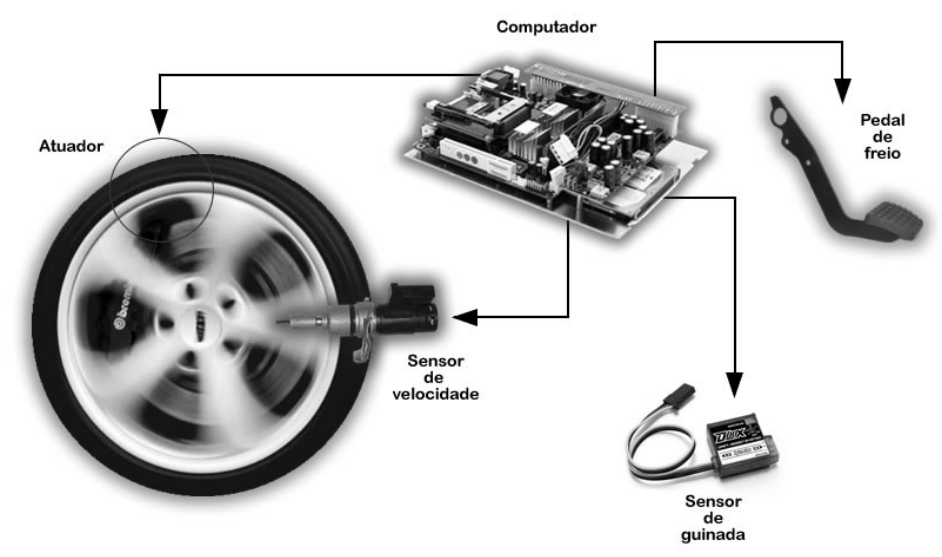

Figura 2 - Arquitetura de um sistema de freios computadorizado

Geralmente, o desenvolvimento de um sistema crítico demanda alto custo, devido às suas características, que são: grande complexidade e dimensão, concorrência ou simultaneidade nas ações, uso de dispositivos de entrada e saída especiais, segurança, confiabilidade e determinismo temporal. Contudo, utilizando extensões de aplicações já existentes e ferramentas livres e de código-aberto é possível desenvolver sistemas com baixo custo e ainda garantir desempenho, como é o caso do $\mathrm{SisCl}$.

\section{Aplicação em Dispositivos Móveis}

Um dispositivo móvel é qualquer equipamento ou periférico que possa ser transportado com conteúdo e esteja acessível em qualquer lugar, são exemplos de dispositivos móveis: pagers, telefones celulares, smartphones, PDAs (Assistentes Digitais Pessoais), bem como aparelhos domésticos que também suportem acesso a uma rede, como microondas, geladeiras, e assim por diante (SILVA, 2010).

Um dispositivo móvel constitui-se basicamente de um processador, uma memória volátil (geralmente RAM ou SRAM), um dispositivo de armazenamento (geralmente a memória FLASH é bastante utilizada para esse fim), e interfaces, que se apresentam em diversas dimensões: $101 \times 65,96 \times 65,154 \times 90,320 \times 240$, etc. Contudo, ao contrário de um computador tradicional, os dispositivos móveis, em sua grande maioria, possuem recursos 
computacionais bem mais limitados, e isso torna a especificação/implementação de aplicações desafiadora para este tipo de plataforma.

Felizmente, hoje muitos dispositivos de comunicação portátil, como celulares, apresentam suporte a plataformas de desenvolvimento de aplicações, como: Java e Android. Os dispositivos com suporte a linguagem Java implementam em seus sistemas, uma máquina virtual que roda aplicações JME (Java Micro Edition). O JME oferece um conjunto poderoso de recursos para a implementação de aplicações críticas, incluindo interface com o usuário, segurança robusta e protocolos de comunicação em rede (JOHNSON, 2007). Por exemplo, técnicas de tolerância a falhas, garantindo alta confiabilidade, segurança, disponibilidade e consistência dos dados das aplicações. São exemplos de implementações, dentre outros já mencionados, sistemas bancários, sistemas de controle de tráfego aéreo. O sistema $\mathrm{Sis} \mathrm{Cl}$, que se trata de um sistema crítico, busca se beneficiar ao máximo desses recursos, como discutido na próxima seção.

\section{SISTEMA SISCI}

O SisCl é um sistema de tempo real para o controle/monitoramento remoto de irrigação(ões) através de dispositivos móveis (celulares). Essa plataforma consiste de (1) uma rede de sensores (ambiente de negócio e obtenção de dados), que são responsáveis por captar as informações de umidade, temperatura, velocidade do vento e pressão atmosférica do campo de irrigação, no intervalo de tempo T e envia esses dados via wireless para (2) uma aplicação Web de gerenciamento orientada a serviços (SisCl - Servidor - ambiente de supervisão e controle), localizada a um raio de 300 metros do campo de irrigação. O SisCl servidor trata de armazenar os dados em um SGBD convencional e disponibilizá-los na Web, eles são usados na geração de gráficos e relatórios. Por fim, há (3) uma aplicação Web JME cliente (Sis Cl - Cliente - ambiente de visualização e controle utilizando dispositivos móveis) pela qual o cliente pode acessar via Internet os dados de seu(s) campo(s) de irrigação(ões) de qualquer lugar do mundo onde ele esteja, isso acessando a página Web disponibilizada pelo SisCl - Servidor ou através da aplicação JME.

Assim, o SisCl apresenta uma junção de características de sistemas de tempo real, com estímulos periódicos e respostas, de sistemas distribuídos (cliente-servidor), onde o servidor possui a prerrogativa de garantir o serviço enquanto o cliente de consumi-lo, e de sistemas orientados a serviços (SOA), onde plataformas e tecnologias de diferentes implementações interoperam (SOMMERVILLE, 2007). A ilustração da Figura 3 expõe em mais detalhes a arquitetura do sistema $\mathrm{SisCl}$.

\section{SisCl Servidor}

O SisCl - Servidor é responsável pelo gerenciamento, tratamento e persistência dos dados no Sistema Gerenciador de Banco de Dados (SGBD) com o fim de gerar gráficos e relatórios com esses dados. No sistema, esses gráficos e relatórios são disponibilizados através de Serviços Web, os quais podem ser acessados diretamente por os usuários ou 
acessados por dispositivos móveis que contenham a versão cliente do $\mathrm{Sis} C \mathrm{Cl}$ (SisCl - Cliente) e que possuam tecnologia necessária para acesso à Internet, como $3 \mathrm{G}$, Wi-Fi, GPRS, entre outros.

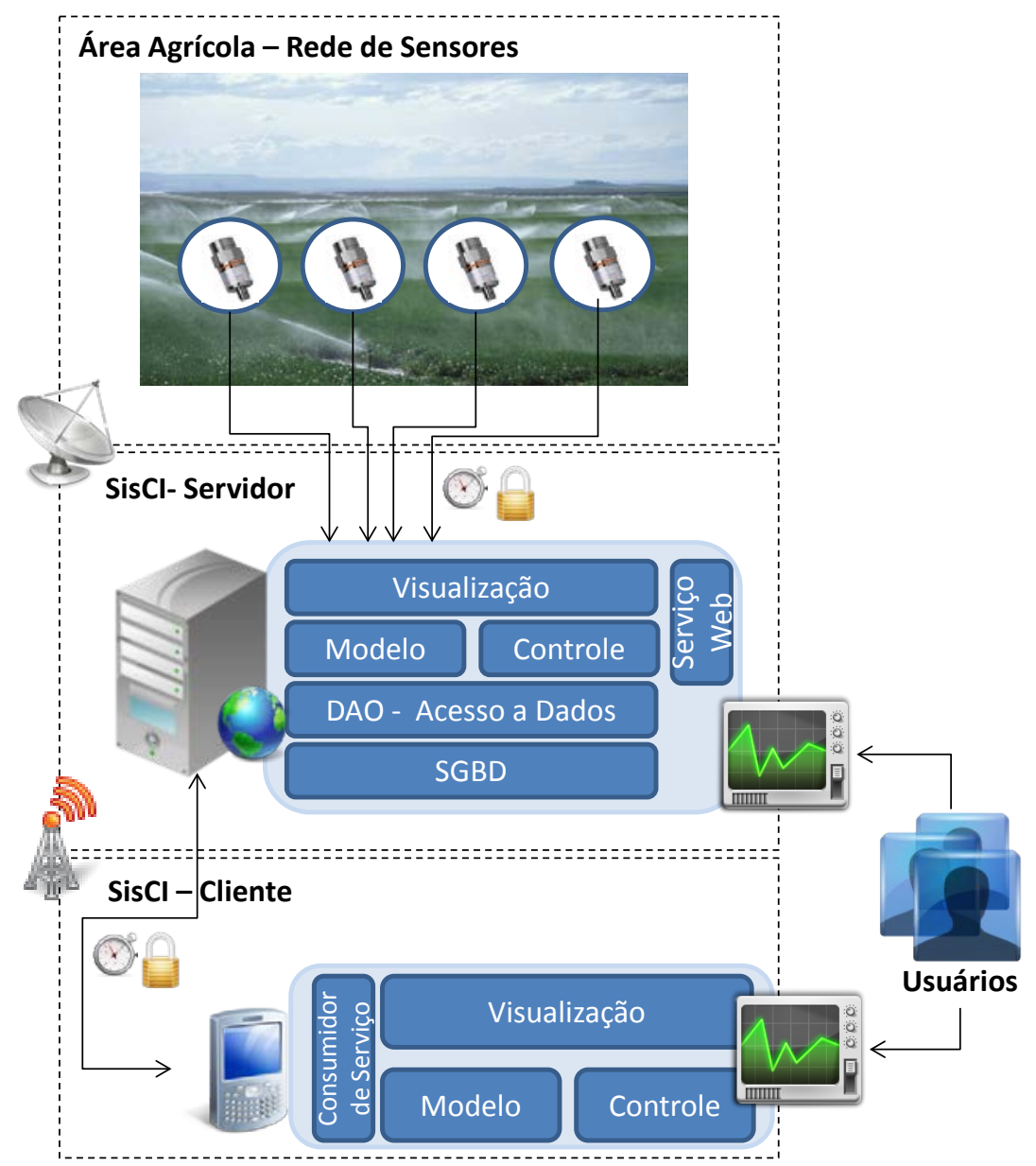

Figura 3 - Arquitetura do sistema $\mathrm{Sis} C \mathrm{I}$

Ao disponibilizar as informações a partir de serviços na Web, o SisCl também assume características da arquitetura de sistemas distribuídos cliente-servidor, onde o servidor possui a prerrogativa de garantir o serviço enquanto o cliente de consumi-lo. Em uma arquitetura cliente-servidor, uma aplicação é modelada como um conjunto de serviços fornecidos pelos servidores e um conjunto de clientes que usam esses serviços (SOMMERVILLE, 2007). Consequentemente, ao permitir o consumo desses serviços a partir de outros programas, o $\mathrm{SisCl}$ - Servidor reúne características inerentes às arquiteturas Orientadas a Serviços (SOA), pois permite que plataformas e tecnologias de implementação diferentes possam ser usadas em diferentes partes das empresas para se interoperarem. 


\section{SisCl Cliente}

O SisCl - Cliente é um aplicação livre desenvolvida para dispositivos de comunicação móveis como celulares, utilizando primitivas de gerenciamento temporais. Garante características de sistemas em tempo real admitindo a responsabilidade de requisitar novas informações da aplicação servidora a cada segundo, a partir de uma conexão segura, e disponibilizar os mesmos em um programa específico que executará em uma plataforma distinta em um dispositivo móvel. Através de um dispositivo móvel que contenha a versão cliente do $\mathrm{SisCl}$ ( $\mathrm{SisCl}$ - Cliente) e que possua tecnologia necessária para acesso à internet, como 3G, Wi-Fi, GPRS, entre outros, o cliente pode, por meio da página Web disponibilizada na Internet pelo servidor, ter acesso aos dados pessoais cadastrados no sistema e aos dados de seu(s) campo(s) de irrigação(ões) também previamente cadastrados (gráficos e relatórios).

\section{METODOLOGIA E RESULTADOS}

O projeto $\mathrm{SisCl}$ se deu na cooperação entre os pesquisadores participantes do projeto, os bolsistas e os técnicos da área agrícola com ênfase em irrigação/manejo. Com um enfoque interdisciplinar, o projeto foi desenvolvido em duas grandes etapas: (i) realização de um estudo teórico/prático nas áreas de informática, eletrônica e agroecologia, focando a verificação/necessidade de requisitos para o sistema, entendimento técnico, etc; (ii) especificação e implementação dos sistemas propostos (SisCl - Servidor e SisCl - Cliente).

Para tanto, o sistema SisCl - Servidor (ver Figura 3) foi desenvolvido na linguagem C\# e ASP.NET, utilizando a ferramenta Microsoft Visual Web Developer 2008 Express Edition. Para a captura dos dados da rede de sensores, foi implementado uma aplicação Java que captura os dados e persiste-os no banco de dados SGBD MySQL 5.1. Por último, o SisCl - Cliente (ver Figura 3) foi implementado utilizando a linguagem Java Micro Edition (JME). Além disso, outras ferramentas auxiliaram na especificação/desenvolvimento do projeto como: MySQL Query Browser, Astah Community e Gantt Project.

Como resultados do projeto, o sistema SisCl - Servidor (ver Figura 3) utilizado para gerenciar todos as informações oferece as seguintes funcionalidades:

- Cadastro de usuários: Registro dos dados de um novo usuário, exemplo: Identificador, Tipo de Usuário(Administrador/Comum), Nome, Login e Senha;

- Cadastro de campo de irrigação: Registro dos dados de um novo campo de irrigação, exemplo: Identificador, Proprietário, Descrição e Localização Geográfica;

- Gerenciamento de usuário: Edição, adição e exclusão de dados de usuário;

- Gerenciamento de campo de irrigação: Edição, adição e exclusão de dados de campo de irrigação;

- Controle de Análise Gráfica: Armazenamento e acesso a análises gráficas de velocidade do vento, umidade relativa do ar, temperatura e taxa de chuva, todas em função do tempo;

- Controle e autenticação de usuários (login e senha): com restrição de visualização de páginas; 
- Capturador de dados Wi-Fi/USB: um middleware para capturar, a cada 3 segundos, dados da rede de sensores.

Por exemplo, as figuras 4 e 5 apresentam respectivamente os gráficos de velocidade do vento e umidade relativa do ar de um campo de irrigação gerados pelo SisCl - Servidor, e a aplicação Web (página inicial) também disponibilizada por ele.

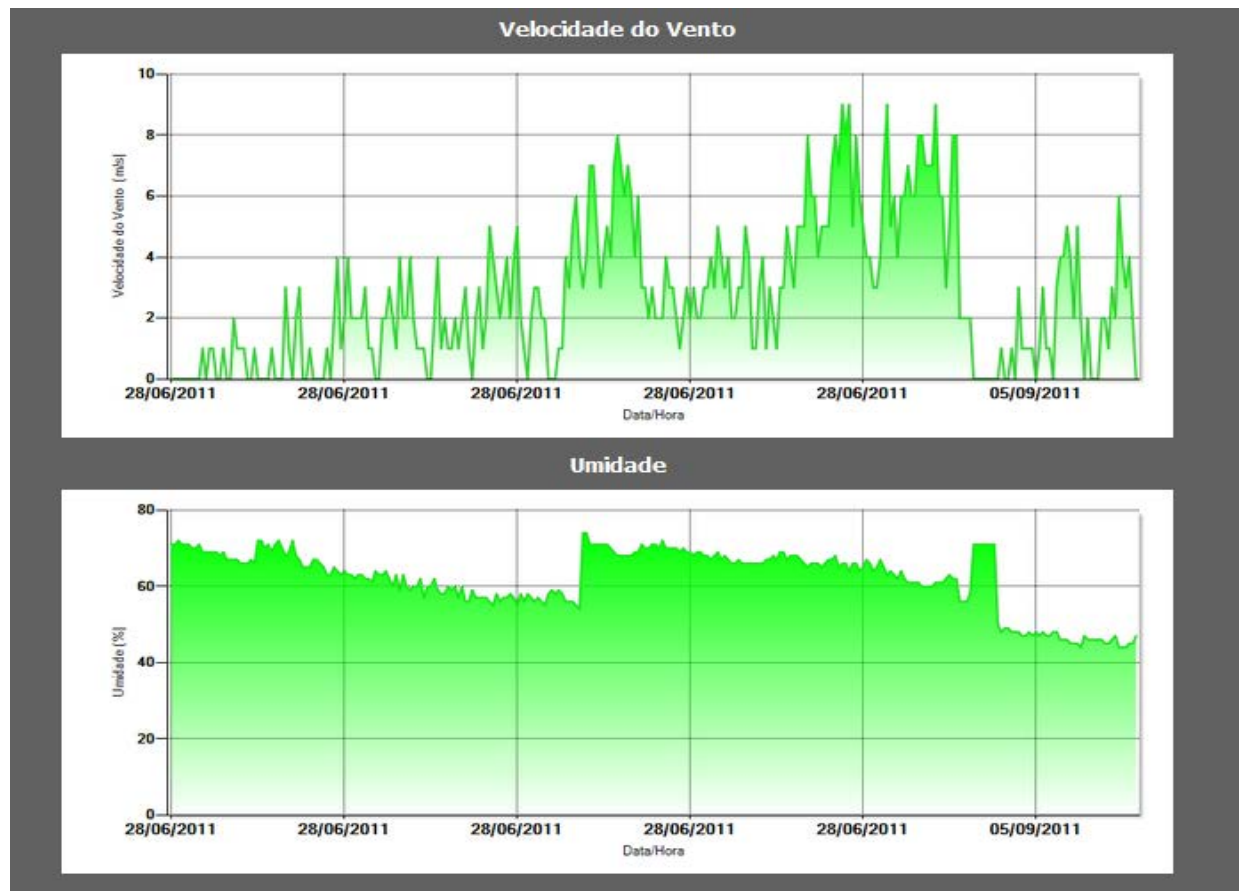

Figura 4 - Gráficos dos dados do Campo de Irrigação gerados pelo SisCl - Servidor

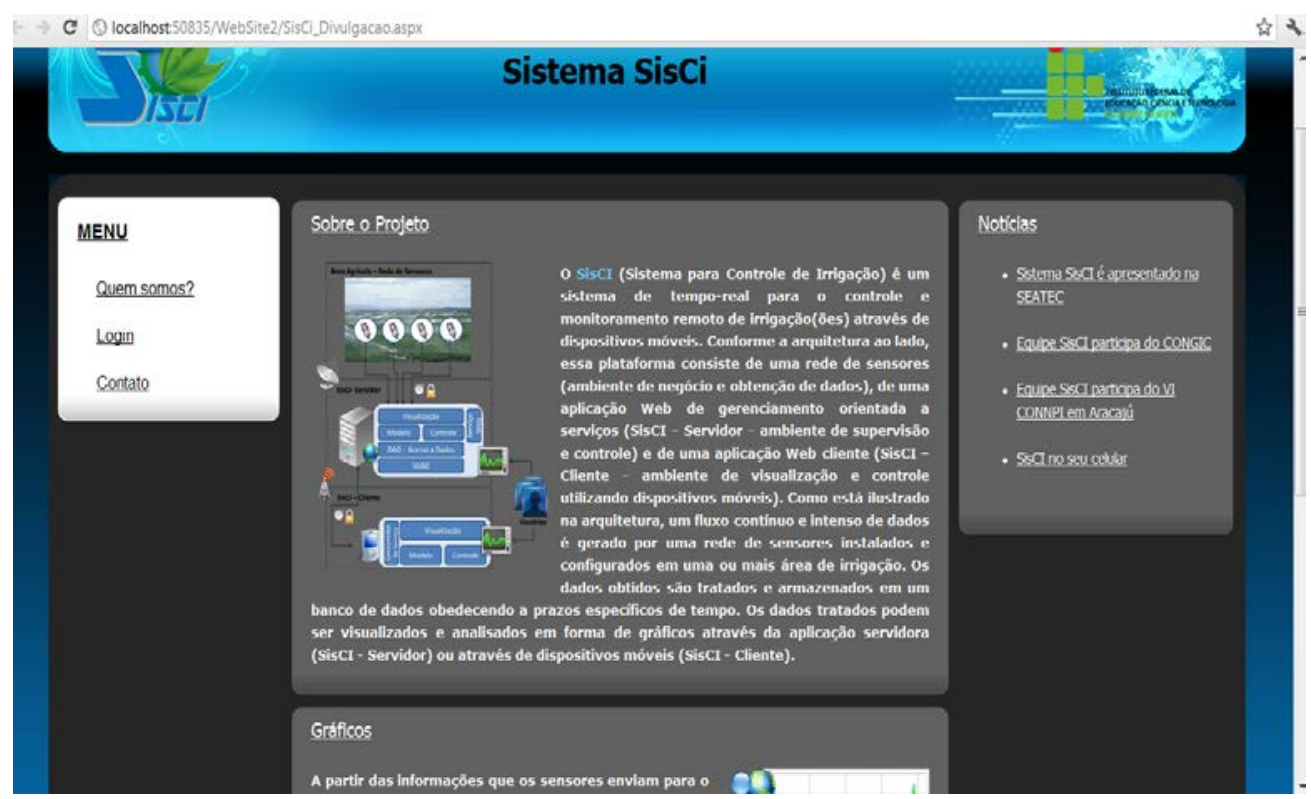

Figura 5 - Aplicação Web Disponibilizada pelo SisCl - Servidor 
Por sua vez, o sistema SisCl - Cliente utilizado para apresentação e análise gráfica dos dados apresenta as seguintes funcionalidades:

- Controle de Análise Gráfica: Armazenamento e acesso a análises gráficas de velocidade do vento, umidade relativa do ar, temperatura e taxa de chuva, todas em função do tempo;

- Controle e autenticação de usuários (login e senha): com restrição de visualização de páginas.

Por exemplo, a Figura 6 apresenta a tela de login (Figura - 6a) e a tela do gráfico da umidade relativa do ar (Figura - 6b) do Sis $\mathrm{Cl}$ - Cliente.

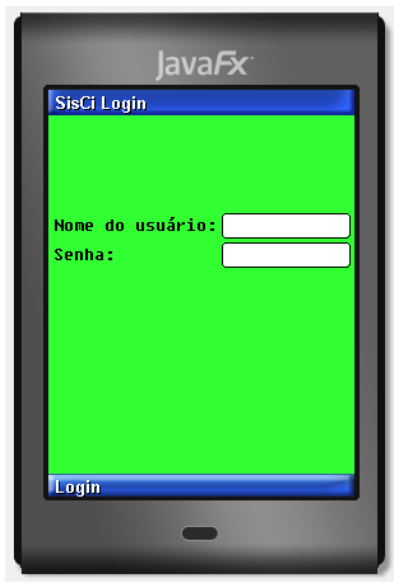

(a)

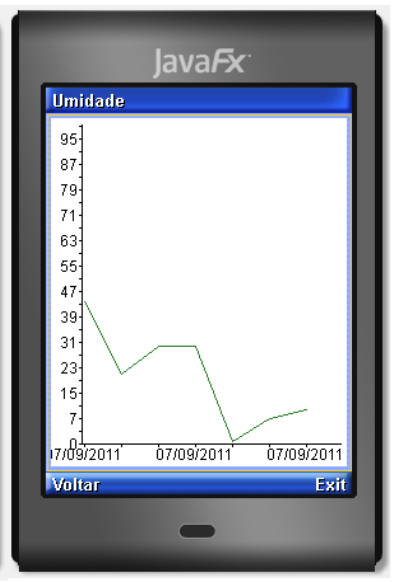

(b)

Figura 6 - Telas da aplicação SisCl - Cliente

\section{CONCLUSÃO}

Neste artigo foi apresentado o sistema SisCl, um sistema de tempo real para o monitoramento remoto de campos de irrigações através de dispositivos móveis. O sistema além de priorizar os softwares livres e de código aberto, concilia ainda, de forma até então não vista na região, conhecimentos e tecnologias pertinentes às áreas de Informática, Agroecologia e Eletrônica. Assim, o projeto promove uma interdisciplinaridade valorosíssima para o enriquecimento profissional/pessoal de pesquisadores, bolsistas e voluntários envolvidos no projeto. Por fim, o projeto encontra-se na fase de implantação e alguns experimentos já realizados, como por exemplo, a geração de relatórios, não apresentados aqui neste artigo, mostram a eficiência e eficácia do SisCl.

Durante o desenvolvimento deste trabalho foram identificados alguns aspectos para trabalhos futuros, entre eles estão: (i) construção de um sistema atuador para junto ao SisCl poder atuar pro-ativamente no ambiente monitorado; (ii) realização de experimentos reais de campo em diversas áreas agrícolas; e, (iii) implementação de técnicas de data mining sobre os dados coletados para alcançar novas concepções. 


\section{REFERÊNCIAS}

1. AUGUSTO, J.A.S. Introdução Sensores Básicos e Princípios de Funcionamento. Faculdade de Lisboa - Portugal, 2006.

2. COSTA, C.M., LEITE, C.R.M., RIBEIRO NETO, P.F. AMSO: Automated System for Monitoring in Real-time Oil Wells On-shores using Sensors Network. International Congress on Engineering and Computer Education - ICECE, Buenos Aires, Argentina, 2009.

3. COSTA, C.M., LEITE, C.R.M., RIBEIRO NETO, P.F. Desenvolvimento de um componente de software - SAMS: Sistema Automático de Monitoramento em Tempo-Real de Sondas de Produção de Petróleo. VIII Conferência Internacional de Aplicações Industriais - INDUSCON, Poços de Caldas, Minas Gerais, 2008.

4. DONG, J., HAI-PING, W. Disaster Recovery Project for Short Message of Personal Access Phone System. Journal of Liaoning University of Petroleum \& Chemical Technology, 2006.

5. JOHNSON, T.M. Java para dispositivos móveis - Desenvolvendo aplicações com j2me. 1a Edição, Novatec, ISBN 978-85-7522-143-3, 2007, p. 336, 2007.

6. MARTINS FILHO, L.C. CIC 180 - Controle de Processos por Computador. Universidade Federal de Ouro Preto - Ouro Preto, 2003.

7. RIBEIRO NETO, P.F ET AL. Uma aplicação de bancos de dados em tempo-real para redes de sensores. VI Workshop de Tempo Real (WTR) - SBRC, p. 45-52, 2004.

8. SALIM, D., BATISTA, T.V., MEDEIROS, A.A.D. A CORBA - Based System for the Monitoring of Petroleum Fields. OTM Workshops, p.34-35, 2004.

9. SHAW, A.C. Sistemas e software de tempo real. trad. Porto Alegre, Bookman, 2003.

10. SILVA, M.G.M., CONSOLO, A.T. Uso de dispositivos móveis na educação - o SMS como auxiliar na mediação pedagógica de cursos a distância. Disponível em: www.5e.com.br/infodesign/146/Dispositivos_moveis.pdf. Acesso em: 23 de novembro de 2010.

11. SOMMERVILLE, I. Engenharia de Software. 8a Edição. Pearson Addison-Wesley, ISBN 978-85-88639-28-7, 2007.

12. SOUZA, R. B., MEDEIROS, A. A. D., NASCIMENTO, J. M. A., MAITELLI, A. L., GOMES, H. P. SISAL - Um Sistema Supervisório para Elevação Artificial de Petróleo. Rio Oil \& Gas Expo and Conference, 2006.

13. SZAMES, Richard. Audioconferência acompanha crescimento mundial da telefonia móvel. Disponível em:

http://www.tinews.com.br/news/2010/04/08/audioconferencia-acompanhacrescimento-mundial-da-telefonia-movel. Acesso em: 11 de janeiro de 2011.

14. WEI-MIN, W., LIU. Design and Implementation of Short Message Service Platform System. Journal of Liaoning University of Petroleum \& Chemical Technology, 2006. 